which formed so striking a feature in this poor woman's case. The very general pleuritic adhesion and thickening of the one side and the immunity of the other is difficult of explanation; for, though the morbid growth had extended principally towards the right, it crossed to the left of the sternum, and the large vessels of the left side were involved; so that we might naturally have looked for a corresponding implication of the left pleura in that inflammatory action which leads to adhesions. I am disposed to think that there had been some old pleuritic affection of the right side, independent of, or accidental to, the morbid growth. But, however this may be, the case shows how different may be the condition of the lung even in protracted cases, and how much the physical signs may thus be modified.

\section{ON THE NATURE OF THE CONDITION CALLED EPILEPSY.}

By J. THOMPSON DICKSON, M.A., M.B.Cantab., M.R.C.P., Medical Superintendent of St. Luke's Hospital

THERE are, perhaps, few subjects upon which the consideration of the physician has been concentrated, which are apparently more obscure than the proximate cause of that mobile condition of the nervous system called epilepsy; while it is certain that within the whole range of medicine, no class of disease has been subjected to the tentative method of treatment with a more decidedly negative result. It has, however, occurred to me that the origin of this state is not really shrouded in such mystery as at first glance appears; and that by a careful study of the evidence which Nature has tendered, we may learn to read her aright in this as we have done in other pathologies.

The first real step in advancing our knowledge of the subject was made by Schroeder van der Kolk, whose researches were followed by those of Brown-Séquard. To these we must add the careful observation of the illustrious Trousseau ; and since, a number of eminent and able practical observers, both on the Continent and in our own country, have added greatly to our store of learning on the subject. It is, however, not my intention in the present paper, to reproduce what has been done, except in so far as quotation may be necessary for the illustration of the ideas herein to be put forward. I may state in the onset that I have repeated many of Brown-Sequard's experiments, and that I have instituted independent ones. It is, however, unnecessary and wearisome to restate burdensome details, and I propose rather to generalise the induced conclusions.

The following are the ideas which it is my intention to attempt to establish.

I. Epilepsy is a contraction of the cerebral capillaries and small arterial vessels; the order of its stages in an epileptic attack being, irritation of brain, either direct or secondary to exhaustion; contraction of cerebral capillaries and small arterial vessels; cerebral anæmia, and consequent loss of consciousness.

2. The muscular contraction and spasm, together with all the varying phenomena associated with epilepsy, are altogether secondary, and not at all essential or constant, but they are all manifestations of im. perfect nervous (cerebral) control, or a loss of balance between the nervous and other systems.

It is perhaps unnecessary to prove loss of consciousness as the first subjective pheromenon of epilepsy, since it is on all hands admitted; or, as stated by Trousseau, it may be considered the pathognomonic sign of epilepsy. To whichever variety of the two great specific forms -le petit mal and le haut mal-any individual seizure may belong, we can always, by strict inquiry, find some amount of unconsciousness in the first stage. The absolute fixedness of this rule-which may almost be called a law of epilepsy-has been doubted, I know, by few. I shall endeavour to meet their objections further on in this paper.

The condition of anæmia is, strange to say, the one which was long unobserved; and, in fact, only within the last few years has it been noticed at all. The congested state of the vessels of the face and neck attendant upon epilepsy is secondary ; but so much more striking is it, that for long it was the only condition of vascularity observed. The pallor of epilepsy is sometimes of considerable duration; but this is more particularly the case in le petit mal, the almost endless varieties of which were not until lately recognised as epileptic, and, consequently, as they were considered as fainting attacks, the attendant pallor made no impression on observers : usually, bowever-especially in le grand malit is fleeting. The fact of pallor, however, is an indication, though not a certain evidence, of cerebral anæmia. It would appear highly probable that the face and neck sympathise with the internal condition of the skull as regards sanguiniference ; but further evidence is necessary, since, on simple principles of animal mechanics, if one set of vessels be empty another set must be more or less full; and the question might reasonably be asked, why the surface-vessels of the head should not be the ones to take up the opposite condition to that of the cerebral. The question would, however, be specious, since it is the venous rather than the arterial vessels that receive the blood; but this is not altogether absolute. The best evidence that the surface-vessels correspond with those of the cerebral is, that consciousness is lost during the anæmic state, while, in conditions presumably congestive, e.g., the paroxysm of whooping-cough, consciousness never, in the true sense of the expression, disappears.

Animals that have died or been killed during a fit, have always exhibited brains perfectly anæmic, blanched, and bloodless. This is borne witness to by Schroeder van der Kolk, Trousseau, and Brown-Séquard, and has obtained in all my own experiments and observations.

3. Whenever cerebral anæmia is by any means brought about, loss of consciousness is the result; e.g., if pressure be made upon the brain or cerebral membranes of an animal, or if the brain be wounded, anæmia and loss of consciousness instantly result. In the case of an infant among the out-patients of Guy's Hospital, said to be epileptic, I made simple pressure with my finger upon an open fontanel, and produced the whole of the epileptic phenomena perfectly. An animal bled to death passes through all the stages of epilepsy before the final struggle. It is hardly necessary to multiply the evidence on this point. It is essential, however, to demonstrate the fact of the contraction of the capillaries and small arterial vessels as a result of irritation or exhaustion, as it is to this contraction that the anæmia and loss of consciousness are due.

Direct irritation, such as that already mentioned, viz., pressure on the membranes or a wound of the brain, are always followed by instantaneous contraction of the smaller cerebral vessels; but, at the same time, the capillaries of the medulla oblongata become distended. This point is one particularly worthy of notice, and was first observed by Schroeder van der Kolk. If an animal be trephined, and a knife plunged into his cerebrum, the whole of that organ will become instantly anæmic, and its small vessels will be found contracted, while those of the medulla - particularly the capillaries-will be found full and distended. It is well to try this experiment on an animal, the subject of epilepsy, as the constant recurrence of the seizures permanently dilates the capillaries of the medulla. It must not be assumed from this that the blood from the cerebral vessels passes to the medulla. It is more likely that, on account of the sudden check to the circulation of blood in the cerebrum, the cumulative force of the arterial current endeavours to expend itself in the nearest channels, of which the medulla forms one; and the absence of resistance, owing to the yielding nature of its material, readily allows the dilatation, which continuously increases the longer the epilepsy is continued.

It thus is clear that, in the relations of cause and effect, the dilatation of the capillaries must be included under the latter head, and must be considered as altogether secondary to the epilepsy.

As the actual pathological lesions associated with epilepsy, I may enumerate tumours involving surface, surface-abscess, tuberculous membranes, thickened membranes, adherent membranes, and atrophy, to which I may add, as a rare though occasional condition, surface-softening, and perhaps softening of the cord.* This synopsis is from an examination of the daily records of post mortem examinations at Guy's Hospital, extending over ten years; and to it I will add bony tumours projecting from the inner table of the skull and encroaching upon the surface of the brain; also ossific membranes.

Extensive disease may occur in the centre of the brain ; but, unless the surface be involved, the central pathology will not be associated with epilepsy as a concomitant.

The histories of clinical cases give unequivocal evidences of tumour and syphiloma, thickening of the cerebral membranes from alcoholism and blows, and hereditary transmission of both syphilis and nervous imperfection; while fright has sometimes been set down as a cause. It occasionally happens, however, that the physician will be baffled in every attempt to find out the particular predisposing cause in an individual.

I have already observed that any cause which tends to produce an anæmic condition of the brain is sufficient to induce convulsion, exampled in the sudden and direct depletion, as when an animal is bled to death : a more gradual drain, however, will produce the same result. For instance, menorrhagia may stand in the relation of cause to effect ; also watery conditions of the blood, as in albuminuria, whether of morbus Brightii or of parturient women. Again, excess of urea, as well as poisons directly introduced into the blood, as atropine, narcotine,

* Among records of post mortem examinations at Guy's Hospital, is one of a case of epilepsy, in which the only lesion noticed was softening of the cord. It is highly probable, however, that this softening was extending to the cerebral surface at the time the patient died. There was no other explanation of the epilepsy in the case. 
nicotine, will also stand in the some relation. Another and not uncommon cause is distant local hyperæmia, exampled especially in children who suffer from convulsive disease, as an affection secondary to disturbance of the digestive organs and the irritation of worms; exempli. fied also in the dentition of infancy; but in this latter case, as perhaps also in a minor degree in the former, the element of peripheral irritation must be somewhat taken into account. Irritation of the peripheral extremity of a nerve will produce epilepsy. A remarkable case was quoted by Dr. Brown-Séquard (reported by the late Mr. Standist of Taunton) and $I$ have seen more than one case of epilepsy traceable to carious teeth. The constant irritation of the extremity of a nerve exhausts the potential energy of the cells from which the nerve takes its rise, and produces a condition very similar to shock, whether physical or psychical. All forms of shock appear undoubtedly to determine the occurrence of epilepsy in some individuals ; yet, with regard to the psychical variety, I would speak very guardedly. One case under my observation for some time was attributed to fright! A post mortem examination exhibited a surface-tumour. Another patient, the widow of an officer who was murdered before her eyes in the Indian mutiny, stated that she had been epileptic ever since the fright she received on that heart-rending occasion. She had, however, suffered from that time up to the time I saw her more or less from menorrhagia. The epilepsy was always in creased when the flux was augmented, and lessened when it abated. After a short treatment, directed towards the control of the menstrual discharge, the epilepsy ceased to recur, and she has remained free from the attack ever since.

Dr. Brown-Séquard, in the commencement of 1869 , stated to the French Academy that, in continuation of his experiments in inducing epilepsy by section of the spinal cord, he had concluded that the greater part of the cord takes an active part in the production of convulsion, because be had seen attacks occur in the muscles innervated by a seg. ment of the cord comprised between two sections. The fact, as stated by Dr. Brown-Séquard, has, however, very little real bearing on the subject of epilepsy, except to confirm the truth of the hypothesis that the convulsive movements of epilepsy are the result of loss of control.

Dr. Brown-Séquard further stated that the brain seemed to take no part in the convulsion, because convulsive seizures continued to be produced in epileptic guinea-pigs, in which life was maintained by artificial respiration after the brain had been removed. This fact at least tends to confirm the idea I proposed at the commencement of this paper; viz., that convulsive movement in epilepsy is the result of a loss of cerebral control, or loss of balance of control between the cerebral and other systems. An animal with a bloodless brain - the effect either of direct depletion or of irritation from any cause-is in very much the same condition as an animal without a brain at all ; and, therefore, there is not that discordance between clinical observation and the results of the experiments of Dr. Brown-Séquard which MM. Colin, Ricord, and Hardy, attempted to show, when Dr. Brown-Séquard's paper was discussed. Dr. Brown-Séquard stated that he had never been able to produce epilepsy by unilateral division of the cord in any animal other than the guinea-pig, except the cat. It is a fact worthy of record that I have induced epilepsy in the rabbit by unilateral section; I have also seen one case of epilepsy in a wild rabbit.

[To be concluded.]

\section{NOTE OF A CASE IN WHICH SEVERE CONSTITU. TIONAL EFFECTS FOLLOWED THE APPLI- CATION OF CANTHARIDINE BLISTER.}

BY JOHN A. CAMPBELL, M.D.,

Assistant Medical Superintendent, Garlands Asylum, near Carlisle.

ON March 15th, 1870, P. D., one of the Asylum officials, a middle-aged man, 16 stone in weight and 5 feet ro inches in height, had been for some time troubled by persistent chronic bronchitis. I applied liquor vesicatorius to the upper part of his chest, 5 inches by 5 inches on each side of his sternum, at 3 P.M.; and told him to put on a linseed-meal poultice over the blistered part at 6 P.M. At 9 P.M., he was seen by Dr. Clouston and myself. We found him sitting on the night-stool, perspiring profusely, looking most haggard and exhausted, depressed in mind, and evidently in great pain. He said that shortly after the linseed-meal poultice had been put on, the blistered part ceased smarting; but that at 7 P.M. he had been taken very ill, that he had to get up to stool, and had been sitting there, straining himself, ever since; that he had not allowed a doctor to be sent for, because he thought a man ought to be able to perform the calls of nature without medical aid. He had passed a little freces and bloody slime, and a little urine.
He complained of great pain at the root of his penis and in his rectum, and said that the few drops of urine he passed felt as if they burned him. He was looking very ill; his pulse was 96 and very weak, and he seemed very faint. We had the blistered surface sponged with warm water to prevent the absorption of more cantharidine, and gave him a glass of gin in cold water and eight grains of compound ipecacuanha powder. He vomited both immediately. He then had a draught containing fifteen minims of tincture of opium, twenty minims of spirit of chloroform, and four ounces of solution of acetate of ammonia. This he retained, but it seemed to do him no good. We then gave him an enema of hot oil with a drachm of solution of hydrochlorate of morphia and twenty minims of the morphia solution with some gin by mouth. The enema came away at once; but another was given, which had such a good effect on the tenesmus, that the patient was able to leave the night-stool and lie down in bed. The strangury remained as severe as ever. We wished to inject some warm oil into his bladder, but he preferred to bear the pain a little longer. At 12 P.M. he was able to stay in bed, though every now and then he felt the tenesmus. We gave him chlorodyne, which made him drowsy and relieved the pain. At 2 A.M. the tenesmus was quite gone, but the strangury continued. We gave him another half a drachm of chlorodyne. At 5 A.M. he fell asleep. When he awoke, he had a great feeling of soreness about the root of the penis and rectum. He was ordered to drink large quantities of barley-water. His urine was examined, but, with the exception of containing a very large quantity of urates, it was normal. The soreness about the rectum and penis continued for two days, and the first time he went to stool he experienced severe pain in the rectum, which remained for several hours. I advised him to take a warm water enema on the next occasion, which he attempted, but found the rectum too tender; so he had to take castor-oil every second day for some time to enable him to have a comfortable passage.

April 28th. - He was now quite recovered, the blister having had a very good effect on his bronchitis, though otherwise it acted un. pleasantly.

REMARKS. - I. This was clearly a case of idiosyncrasy, rendering the patient most susceptible to the effects of cantharides; for, though cases occasionally occur where slight tenesmus and strangury follow the application of a cantharides blister, I have never heard of such results following the application of the liquid preparations. The preparation used was Burt's liquor vesicatorius. Fluid out of the same bottle has been used repeatedly by me before and since its application in this case, without producing any but the desired effect. 2. The mental depression, noticed by writers on the toxic effects of cantharides taken by the mouth, was strongly marked. The patient, an unusually sensible, courageous, and determined man, could not control his expressions of despondency and pain and kept moaning most of the time, and saying he felt as if he could lie down on the floor and die. The patient had no feeling of priapism. 3. The warm enemata and the chlorodyne seemed to give the most relief.

\section{CASE OF VARICELLA IN AN ADULT.}

By W. T. GREENE, M.B., etc.

ON January 30 th, 1868 , an adult, aged 32 , was exposed to the infection of varicella for several hours. On February 7 th, he began to feel unwell, and thought he was getting a cold, to attaks of which he was subject. On February 8th, he was much worse, with severe headache; pulse 70. The next day the headache and prostration had much increased; there was also considerable nausea, and slight rigors. Two or three small vesicles appeared on the chest, towards evening. On the Ioth, a number of vesicles appeared on the chest, back, shoulders, arms, and scalp; they were of different sizes, but clear and prominent, with a small red are la re nausea gone. On the I Ith, more vesicles appeared on the thighs, buttocks, and face, some on the eyelids and on the fauces. On the I 2 th, he felt quite well, but a few more vesicles came out on the legs, one on the glans penis, and two or three on the corona glandis; there was considerable pruritus from this date for several days. On the 13 th, one or two papulæ made their appearance on the hands, but no more vesicles; the others also assumed a pustular appearance, and a few of then dried up. In a fortnight from the commencement of the attack, almost all trace of the eruption had disappeared. On puncturing one or two of the larger vesicles with a needle, in their first stage, the contents, and colourless fluid, were entirely evacuated.

Exactly one fortnight from the commencement of the attack, or one week after it had reached its height, an infant in the house sickened, the eruption appeared on the third day, and ran its usual course.? 